\title{
Half-cycle optical soliton in quadratic nonlinear media
}

\author{
Hervé Leblond \\ Laboratoire POMA, FRE 2988, Université d'Angers, 2 Boulevard Lavoisier, 49000 Angers, France
}

(Received 9 November 2007; revised manuscript received 30 April 2008; published 8 July 2008)

\begin{abstract}
We show that a few-cycle pulse launched in a quadratic medium may result in a half-cycle soliton in the form of a single hump, with no oscillating tail. The analysis involves the derivation of a Korteweg-de Vries $(\mathrm{KdV})$ equation from both a classical and a quantum mechanical simple model of matter-radiation interaction. The sign of the electric field in the half-cycle $\mathrm{KdV}$ soliton is fully determined by the properties of the material, which definitely breaks the phase invariance.
\end{abstract}

DOI: 10.1103/PhysRevA.78.013807

PACS number(s): 42.65.Tg, 42.65.Re

\section{INTRODUCTION}

Ultrafast optics has led recently to the production of pulses with durations down to a few optical cycles (see the reviews $[1,2])$. The theoretical study of few-cycle pulses (FCPs) propagation is the matter of extensive research: corrections to the paraxial approximation [1-3], the so-called short-pulse equation (SPE) [4-6]. Self-compression of the FCP and FCP soliton formation is a very important issue [7]. The propagation of FCP solitons in Kerr media can be described in a way completely free from the slowly varying envelope approximation, using completely integrable models as the modified Korteweg-de Vries (mKdV), sine-Gordon (sG) or $\mathrm{mKdV}$-sG equations [8-10].

However, a FCP still remains a wave packet, which presents a large number of oscillations, even if only one or two of them have an important amplitude. A challenge is to produce an optical pulse with a single oscillation, comparable in shape with the solitary waves which are well known in hydrodynamics; in other words, to clean up the FCP by suppressing the oscillations around the main hump.

A second point is the phase invariance, which may appear as a fundamental symmetry of the optics. The FCP in some sense loses the phase invariance, and the importance of the carrier-envelope (CE) phase has been emphasized [1]. However, although ways of stabilizing the CE phase have been proposed $[10,11]$, it remains a very difficult task. Further, even if a zero CE phase is realized, the existing setups cannot distinguish it from a phase $\pi$ : the polarity of the electric field remains random, and its mean value on a large number of FCPs remains zero.

Up to now, the study of FCP solitons is restricted to the case of a cubic (Kerr) nonlinearity. For longer pulse durations, when the slowly varying envelope approximation is valid, the study of optical solitons in quadratic nonlinear media has shown many remarkable properties with respect to the cubic Kerr case $[12,13]$. First of all, the quadratic nonlinearity, in principle, allows one to observe nonlinear effects with much lower input power than a cubic one. However, such an effect and the formation of envelope solitons in quadratic media, involve at least two components, a fundamental frequency and a second harmonic [14]. The interaction between the two components is efficient if some phasematching condition is satisfied; experimentally it can be achieved by different ways, e.g., by temperature adjustment
[15]. It has been shown that a result of this interaction is the suppression of the collapse, which occurs in two-dimensions in a Kerr medium, leading to the formation of twodimensional solitons [16], and to the stabilization of many types of spatial and spatiotemporal solitons [17]. Far from the phase-matching, the so-called cascading effect leads to an effective cubic nonlinearity [18]. However, selfrectification and electro-optic effect remain, which are able to stop the collapse [19], and also can be used to control the pulse by means of adequately matched microwaves [20]. The question, whether and how these properties might generalize to FCPs, naturally arises, and in a first stage, it is worth investigating the possibility to build a theory for FCP soliton propagation in quadratic media.

We show in the present paper that launching a FCP into a medium with an electronic quadratic nonlinearity allows one to produce half-cycle pulses, with no oscillating tail, a perfectly zero CE phase, and a definite polarity of the field. The process breaks the phase invariance, even statistically, and yields an optical electric field with definite polarity, and nonzero mean value.

Our approach is based on the soliton theory. Indeed, solitons, i.e., fundamental solutions of integrable equations as Korteweg-de Vries (KdV), mKdV, or sG involve solitary waves, i.e., single-oscillation, half-cycle pulses. Each pulse of this kind has a determined sign or polarity. Hence, due to phase invariance, it is believed that such pulses cannot be produced by an optical system, at least in the present state of knowledge. Breather solutions of $\mathrm{mKdV}$, sG, and $\mathrm{mKdV}$-sG have been given by the mathematical theory; they do not involve such a symmetry breaking. It has been shown that such objects were good candidates for the description of FCPs $[9,10]$. We prove in the present paper that FCP soliton propagation in a quadratic medium can be described by the $\mathrm{KdV}$ equation itself, which is quadratic, and not by the cubic $\mathrm{mKdV}$ equation. Note that all solitons of KdV have the same sign, while $\mathrm{mKdV}$ admits both positive and negative solitons. Further KdV does not present breather solutions, and it will be shown that the KdV soliton itself, a half-cycle pulse, will arise from the propagation of an input FCP with mean value zero. The symmetry breaking in the wave, related to the existence or nonexistence of a breather, originates in the noncentrosymmetry of the quadratic medium. 


\section{KORTEWEG-DE VRIES MODEL FOR QUADRATIC FCP SOLITONS}

\section{A. Classical approach}

\section{Derivation of $\mathrm{KdV}$}

We consider first a classical model of an elastically bounded electron oscillating along the polarization direction $x$ of the wave. The latter propagates in a direction $z$ perpendicular to $x$, in such a way that the transverse variations can be neglected. The evolution of the position $x$ of an atomic electron is described by an anharmonic oscillator (damping is neglected)

$$
\frac{d^{2} x}{d t^{2}}+\Omega^{2} x+a x^{2}=\frac{-e}{m} E,
$$

where $\Omega$ is the resonance frequency, $-e$ is the electron charge, $m$ is its mass, and $E$ is the wave electric field component along $x$. The evolution of $E$ is described by the Maxwell equations, which reduce to

$$
\partial_{z}^{2} E=\frac{1}{c^{2}} \partial_{t}^{2}(E+4 \pi P),
$$

where $P=-$ Nex is the polarization density, $N$ being the density of atoms.

Transparency implies that the characteristic frequency $\omega_{w}$ of the considered radiation (in the optical range) strongly differs from the resonance frequency $\Omega$ of the atoms; hence it can be much higher or much lower. We consider here the latter case, i.e., we assume that $\omega_{w}$ is much smaller than $\Omega$. This motivates the introduction of the slow variables

$$
\tau=\varepsilon\left(t-\frac{z}{V}\right), \quad \zeta=\varepsilon^{3} z
$$

$\varepsilon$ being a small parameter. The delayed time $\tau$ involves propagation at some speed $V$ to be determined. It is assumed to vary slowly in time, according to the assumption $\omega_{w} \ll \Omega$. The pulse shape described by the variable $\tau$ is expected to evolve slowly in time, the corresponding scale being that of variable $\zeta$.

A weak amplitude assumption is needed in order that the nonlinear effects arise at the same propagation distance scale as the dispersion does. Hence we expand

$$
E=\varepsilon^{2} E_{2}+\varepsilon^{3} E_{3}+\varepsilon^{4} E_{4}+\cdots,
$$

as in the standard $\mathrm{KdV}$-type expansions [21]. $P$ is expanded in the same way. The leading order, i.e., Eq. (1) at order $\varepsilon^{2}$ and Eq. (2) at order $\varepsilon^{4}$, gives

$$
P_{2}=\frac{N e^{2}}{m \Omega^{2}} E_{2}
$$

and

$$
V=c\left(1+\frac{4 \pi N e^{2}}{m \Omega^{2}}\right)^{-1 / 2} .
$$

Then it is seen that $E_{3}$ can be taken as zero. At next order $\left[\varepsilon^{4}\right.$ in Eq. (1)], we get

$$
P_{4}=\frac{N e^{2}}{m \Omega^{2}} E_{4}-\frac{N e^{2}}{m \Omega^{4}} \partial_{t}^{2} E_{2}+\frac{a N e^{3}}{m^{2} \Omega^{6}}\left(E_{2}\right)^{2} .
$$

Reporting into Eq. (2) at order $\varepsilon^{6}$, the terms in $E_{4}$ cancel using Eq. (6), and we obtain the $\mathrm{KdV}$ equation

$$
\partial_{\zeta} E_{2}=A \partial_{\tau}^{3} E_{2}+B \partial_{\tau}\left(E_{2}\right)^{2},
$$

with the coefficients

$$
A=\frac{4 \pi V N e^{2}}{2 m c^{2} \Omega^{4}}, \quad B=\frac{-4 \pi V a N e^{3}}{2 m^{2} c^{2} \Omega^{6}},
$$

for the dispersion and nonlinearity, respectively. We emphasize the fact that Eq. (8) is satisfied by the electric field itself, and not by some envelope: there is no carrier. It is important to notice that, since Eq. (8) is not an envelope equation, the third order time derivative does not represent a third order dispersion. As shown in Ref. [22], it includes at least the second and third order dispersions.

\section{Coefficients of $K d V$}

The dispersion relation of model (1-2) is well known and easily computed. The refractive index is

$$
n=\left(1+\frac{4 \pi N e^{2}}{m} \frac{1}{\Omega^{2}-\omega^{2}}\right)^{1 / 2} .
$$

We check that $V=c / n$. Defining the wave vector by $k$ $=\omega n / c$, we check by straightforward computation that the dispersion coefficient is

$$
A=\left.\frac{1}{6} \frac{d^{3} k}{d \omega^{3}}\right|_{\omega=0}=\left.\frac{1}{2 c} \frac{d^{2} n}{d \omega^{2}}\right|_{\omega=0} .
$$

Expression (11) is exactly the same as in the case of the $\mathrm{mKdV}$ model accounting for FCP propagation in a Kerr medium [9]. Notice that, due to the long-wave approximation, the dispersion coefficient $A$ only involves the second derivative of $n$, as the second order dispersion $d^{2} k / d \omega^{2}(\omega)$ in the case of envelope solitons. The nonlinear susceptibility corresponding to model (1-2) reads as [23]

$$
\chi^{(2)}(2 \omega, \omega, \omega)=\frac{a N e^{3}}{m^{2}} \frac{1}{\left(\Omega^{2}-\omega^{2}\right)^{2}\left(\Omega^{2}-4 \omega^{2}\right)}
$$

(recall that the damping is neglected). Hence

$$
B=\left.\frac{-2 \pi}{n c} \chi^{(2)}(2 \omega, \omega, \omega)\right|_{\omega=0} .
$$

It is analogous to the expression obtained for the $\mathrm{mKdV}$ model [9], which involves $\chi^{(3)}(\omega ; \omega, \omega,-\omega)$, and is valid for a cubic Kerr nonlinearity.

\section{B. Quantum mechanical approach}

\section{Second derivation of $K d V$}

We consider a set of two-level atoms with the Hamiltonian 


$$
H_{0}=\hbar\left(\begin{array}{cc}
\omega_{a} & 0 \\
0 & \omega_{b}
\end{array}\right),
$$

where $\Omega=\omega_{b}-\omega_{a}>0$ is the frequency of the transition. The evolution of the electric field $E$ (we restrict to one field component for the sake of simplicity) is described by the wave equation (2). The light propagation is coupled with the material by means of a dipolar electric momentum $\mu$ directed along the same direction $x$ as the electric field, according to

$$
H=H_{0}-\mu E,
$$

and the polarization density

$$
P=N \operatorname{Tr}(\rho \mu)
$$

along the $x$ direction, $N$ being the volume density of atoms, and $\rho$ the matrix density. The latter obeys

$$
i \hbar \partial_{t} \rho=[H, \rho]+\mathcal{R},
$$

where $\mathcal{R}$ is a phenomenological relaxation term. In the case of cubic nonlinearity, it has been shown that it was negligible [9], and it will be neglected here. In the absence of permanent dipolar momentum,

$$
\mu=\left(\begin{array}{cc}
0 & \mu \\
\mu^{*} & 0
\end{array}\right)
$$

is off-diagonal, and the quadratic nonlinearity is zero.

The quadratic nonlinearity can be phenomenologically accounted for as follows: it corresponds to a deformation of the electronic cloud induced by the field $E$, hence to a dependency of the energy of the excited level $b$ with respect to $E$ : a Stark effect. The nonlinearity is quadratic if this dependency is linear, as

$$
\omega_{b} \rightarrow \omega_{b}-\alpha E .
$$

This can be included phenomenologically in the MaxwellBloch equations by replacing the free Hamiltonian $H_{0}$ with

$$
H_{0}-\alpha E\left(\begin{array}{ll}
0 & 0 \\
0 & 1
\end{array}\right) \text {. }
$$

The equations are exactly the same if the dipolar momentum $\mu$ is replaced with

$$
\mu=\left(\begin{array}{cc}
0 & \mu \\
\mu^{*} & \alpha
\end{array}\right),
$$

i.e., Eq. (20) is equivalent to the assumption that the excited state $b$ has a nonzero permanent dipolar momentum $\alpha$.

To simplify computations, we use the same rescaling as in Ref. [9], so that the constants $c, N, \hbar$, and $4 \pi$ are replaced with 1 . Then $\operatorname{Tr}(\rho)$ is not 1 anymore but $\nu=4 \pi N \hbar c$. We denote the elements of a matrix $M$ by indices $a, b, t, u$ according to

$$
M=\left(\begin{array}{ll}
M_{a} & M_{t} \\
M_{u} & M_{b}
\end{array}\right) .
$$

We use the same slow variables and power expansion as for the classical model, with

$$
\rho_{0}=\left(\begin{array}{ll}
\nu & 0 \\
0 & 0
\end{array}\right)
$$

Apart from this term the expansion begins at order $\varepsilon^{2}$.

Equation (17) at order $\varepsilon^{2}$ gives

$$
\rho_{2, t}=\frac{\nu \mu}{\Omega} E_{2},
$$

$\rho_{2, a, b}$ remaining free. We deduce

$$
P_{2}=\frac{2 \nu|\mu|^{2}}{\Omega} E_{2}
$$

Reporting Eq. (25) into Eq. (2) at order $\varepsilon^{4}$, we get the value of the velocity

$$
V=\left(1+\frac{2 \nu|\mu|^{2}}{\Omega}\right)^{-1 / 2} .
$$

The expression of $V$ is exactly the same as in the $\mathrm{mKdV}$ case [9], since the linear part of the model is exactly the same.

At order $\varepsilon^{3}$, Eq. (17) shows that $\rho_{2, a}=\rho_{2, b}=0$ and

$$
\rho_{3, t}=\frac{\nu \mu}{\Omega} E_{3}-\frac{i \nu \mu}{\Omega^{2}} \partial_{\tau} E_{2},
$$

from which we deduce

$$
P_{3}=\frac{2 \nu|\mu|^{2}}{\Omega} E_{3} .
$$

Hence the wave equation (2) at order $\varepsilon^{5}$ does not give any further information.

Equation (17) at order $\varepsilon^{3}$ gives useless expressions involving $\rho_{3, a, b}$ and

$$
\rho_{4, t}=\frac{\nu \mu}{\Omega} E_{4}-\frac{i \nu \mu}{\Omega^{2}} \partial_{\tau} E_{3}-\frac{\nu \mu}{\Omega^{3}} \partial_{\tau}^{2} E_{2}+\frac{\alpha \nu \mu}{\Omega^{2}}\left(E_{2}\right)^{2},
$$

from which we deduce

$$
P_{4}=\frac{2 \nu|\mu|^{2}}{\Omega} E_{4}-\frac{2 \nu|\mu|^{2}}{\Omega^{3}} \partial_{\tau}^{2} E_{2}+\frac{2 \alpha \nu|\mu|^{2}}{\Omega^{2}}\left(E_{2}\right)^{2} .
$$

Reporting Eq. (30) into the wave equation (2) at order $\varepsilon^{6}$ we get, after cancellation of the terms involving $E_{4}$, and one integration with respect to $\tau$ (the field and derivatives are assumed to vanish at infinity),

$$
\partial_{\zeta} E_{2}=\frac{V \nu|\mu|^{2}}{\Omega^{3}} \partial_{t}^{3} E_{2}-\frac{\alpha V \nu|\mu|^{2}}{\Omega^{2}} \partial_{\tau}\left(E_{2}\right)^{2} .
$$

Equation (31) is exactly the KdV equation (8), with the dispersion and nonlinear coefficients (in physical units)

$$
A=\frac{4 \pi N|\mu|^{2}}{n c \hbar \Omega^{3}}, \quad B=\frac{-4 \pi N \alpha|\mu|^{2}}{n c \hbar^{2} \Omega^{2}},
$$

respectively, the refractive index being 


$$
n=\sqrt{1+\frac{8 \pi N|\mu|^{2}}{\hbar \Omega}} .
$$

The dispersion relation is the same as in the Kerr case [9], and the same expression of $A$ still holds; it coincides with Eq. (11).

\section{Coefficients of $K d V$ in the quantum mechanical approach}

The dispersion relation is the same as in the Kerr case [9], and the same expression of $A$ still holds; it coincides with Eq. (11).

The second order susceptibility is computed as follows: we report into Eqs. (15)-(17) the following harmonic electric field excitation,

$$
E=\varepsilon \mathcal{E}\left(e^{i \omega t}+e^{-i \omega t}\right)
$$

and expand the density matrix as

$$
\rho=\rho_{0}+\varepsilon \rho_{1}(t)+\varepsilon^{2} \rho_{2}(t)+\cdots,
$$

with the same $\rho_{0}$ as above. We obtain at first order $\rho_{1, a, b}=0$ and

$$
\rho_{1, t}=C_{+} e^{i \omega t}+C_{-} e^{-i \omega t}
$$

with

$$
C_{ \pm}=\frac{\mu \mathcal{E}}{\hbar(\Omega \mp \omega)} .
$$

At order $\varepsilon^{2}$, Eq. (17) is easily soved to yield $\rho_{2, a, b}=0$ and

$$
\rho_{2, t}=K_{+} e^{2 i \omega t}+K_{-} e^{-2 i \omega t}+K_{0},
$$

with

$$
K_{ \pm}=\frac{\alpha \mu \mathcal{E}^{2}}{\hbar^{2}(\Omega \mp 2 \omega)(\Omega \mp \omega)} .
$$

The second order susceptibility is related to the polarization, though

$$
\begin{aligned}
P & =\varepsilon^{2} \chi^{(2)}(2 \omega ; \omega, \omega) \mathcal{E}^{2} e^{2 i \omega t}+\cdots \\
& =\varepsilon^{2} N \operatorname{Tr}\left(K_{+} \mu^{*}+K_{-}^{*} \mu\right) e^{2 i \omega t}+\cdots
\end{aligned}
$$

and hence

$$
\begin{aligned}
\chi^{(2)}(2 \omega ; \omega, \omega)= & \frac{N \alpha|\mu|^{2} \mathcal{E}^{2}}{\hbar^{2}}\left(\frac{1}{(\Omega+2 \omega)(\Omega+\omega)}\right. \\
& \left.+\frac{1}{(\Omega-2 \omega)(\Omega-\omega)}\right) .
\end{aligned}
$$

The nonlinear coefficient $B$ of the KdV equation expresses as

$$
B=\left.\frac{-2 \pi}{n c} \chi^{(2)}(2 \omega ; \omega, \omega)\right|_{\omega=0} .
$$

We obtain exactly the same expression as for the classical model.

\section{Generalization}

It can be reasonably conjectured that the $\mathrm{KdV}$ model with the expression of the coefficients in terms of the linear re- fractive index and second order susceptibilities is valid in more general and realistic situations than the simple models considered in this paper.

The latter assumes a single resonance line, at some frequency $\Omega$. The long-wave approximation used to derive $\mathrm{KdV}$ assumes that the typical frequency $\omega$, which is comparable to the inverse $\omega \simeq 1 / T$ of the duration $T$ of the solitary wave, is small with respect to $\Omega$. With $\omega$ belonging to the visible range, it means that the resonance line of the material belongs to the uv.

Expression (11) of the dispersion coefficient is expected to generalize as far as $\omega$ is far from any resonance line of the material. The expression (43) of the nonlinear coefficient is valid, in principle, if only one transition in the uv is involved by the quadratic nonlinearity. If several transitions are involved, all nonlinear terms will have the same form as in Eq. (8), and the coefficients will combine together to yield Eq. (43) again. Regarding the transition lines, which are far below $\omega$, it has been seen in the cubic case [9] that they required a much higher power input than the uv transitions to produce an observable nonlinear effect. Hence they are expected to be negligible, at least in certain materials.

The values of the dispersion coefficient and $\chi^{(2)}$ are taken at $\omega=0$. Due to the perturbative approach, this means in fact that they are taken at a value of $\omega$ well below the resonance line $\Omega$, as is assumed to be the inverse $\omega=1 / T$ of the pulse duration, typically in the visible range.

Notice that we have here a pure quadratic nonlinearity for a single wave, and that no effective third order nonlinearity due to cascaded second order ones is involved. This makes a sharp contrast with the nonlinear propagation of parametric solitons within the slowly varying envelope approximation. In particular, no phase matching is required.

\section{HALF-CYCLE QUADRATIC FCP SOLITON}

\section{A. KdV soliton}

The fundamental soliton of $\mathrm{KdV}$ is expressed in the present case as

$$
E_{2}=\frac{k_{3} n c p^{2}}{-2 \pi \chi^{(2)}} \operatorname{sech}^{2}\left(p \tau+\frac{2}{3} k_{3} p^{3} \zeta\right),
$$

where we have set

$$
k_{3}=\left.\frac{d^{3} k}{d \omega^{3}}\right|_{\omega=0}
$$

and

$$
\chi^{(2)}=\left.\chi^{(2)}(2 \omega ; \omega, \omega)\right|_{\omega=0} .
$$

It is shown in Fig. 1(c). It differs from the soliton of $\mathrm{mKdV}$ [Fig. 1(a)], which is a sech and not a $\operatorname{sech}^{2}$. It can be obtained by direct integration [24], by the Hirota method [25], or by the inverse scattering transform (IST) [26,27]. The stability and robustness of the fundamental soliton (44) is ensured from the IST for any positive $p$. The corresponding solutions are proven to be the fundamental modes of the $\mathrm{KdV}$ equation, in the sense that any input decomposes into a combination of a finite number of solitons and Fourier-type 

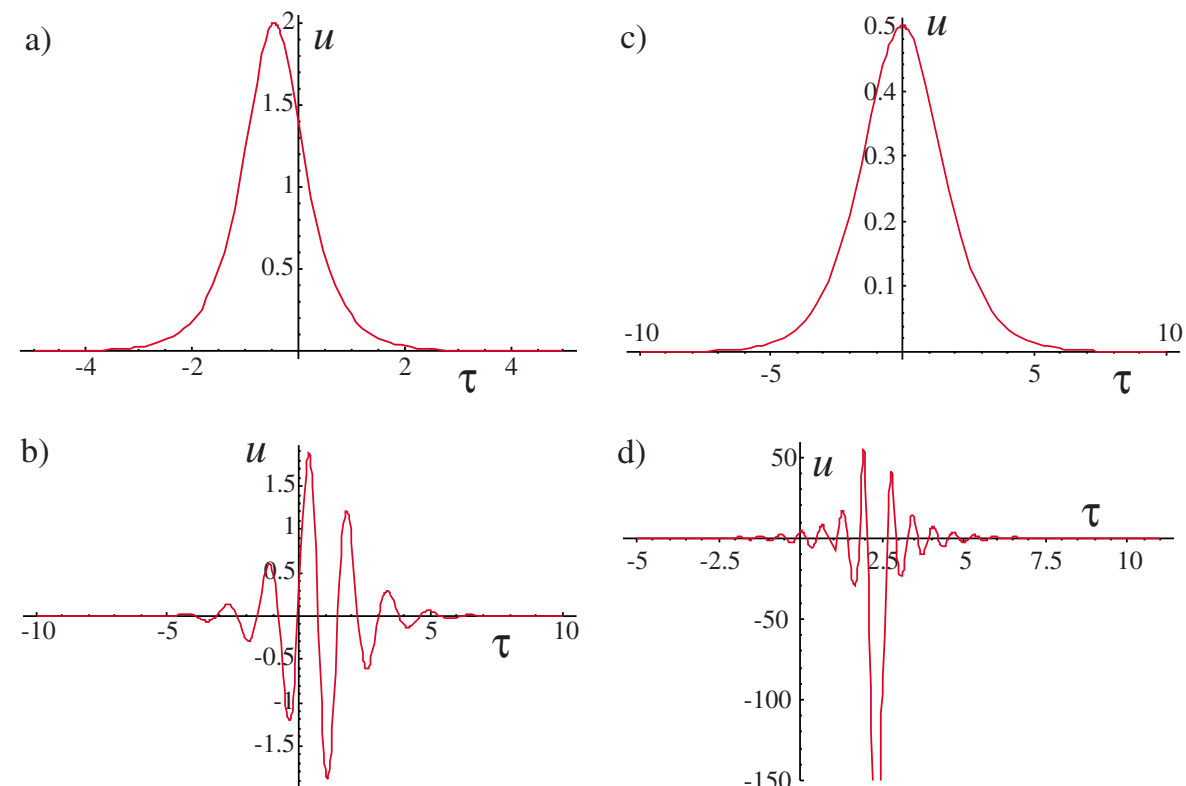

d)

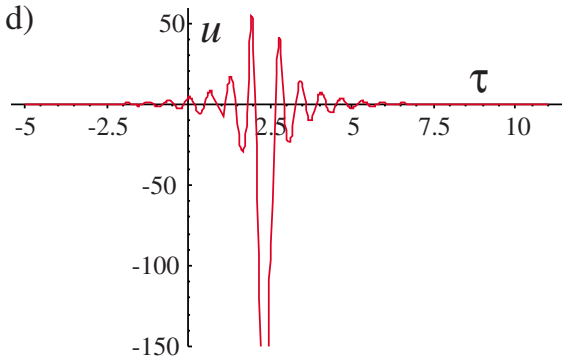

FIG. 1. (Color online) (a) Soliton solution to $\mathrm{mKdV}$. (b) Breather solution to $\mathrm{mKdV}$ : the FCP soliton in Kerr media. (c) Soliton solution to $\mathrm{KdV}$ : the FCP soliton in quadratic media. (d) The breatherlike solution of $\mathrm{KdV}$ : cannot describe a FCP since it is singular. modes called "radiation," which evolve separately, conserving their characteristics all the propagation long.

For the sake of convenience, below we use the standard dimensionless form of the $\mathrm{KdV}$ equation (8),

$$
u_{Z}+6 u u_{\tau}+u_{\tau \tau \tau}=0
$$

which is obtained by means of he linear transform $Z=-A \zeta$, $u=(B / 6 A) E_{2}$. Direct numerical simulation illustrates and confirms the mathematical result: for $\mathrm{KdV}$, a FCP-type input decays into solitons, with a definite sign (Figs. 2 and 3). For a short enough FCP, the number of solitons is only 1 or 2 . In the latter case, the two solitons have different velocities, and are conserved when they interact (Fig. 4). The number of emitted solitons depends on the CE phase of the initial FCP at the entrance of the quadratic medium (see Fig. 5).

However, in every case (i) single-oscillation (half-cycle) pulses are produced and (ii) the sign of $u$, i.e., the sign of the electric field, is always the same. Indeed, the relative sign of $u$ and $E_{2}$ is that of $B / A$ and cannot be modified by a real linear transform. For the two-level model, we have $B / A$

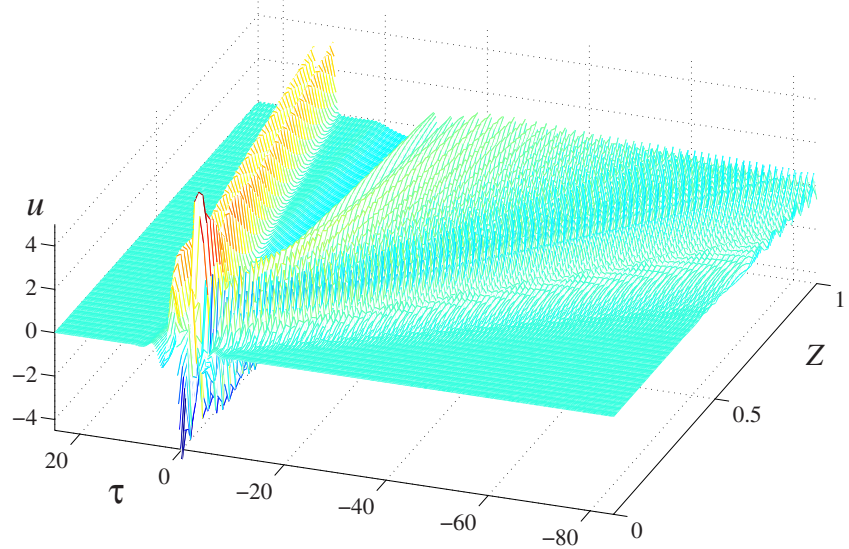

FIG. 2. (Color online) Evolution of an initial FCP into KdV solitons and dispersive waves. $=-\alpha \Omega / \hbar$, i.e., it is proportional to the dipolar momentum of the excited state. In the classical model, we can see that $B / A=-2 a e /\left(m \Omega^{2}\right)$, which has the same physical origin. In the general case $B / A$ involves both the quadratic nonlinearity and the dispersion. Hence the polarity of the optical electric field is fully determined by the direction of the polarization of the atoms, and the dispersion.

\section{B. $m K d V$ equation possesses breathers while $\mathrm{KdV}$ does not}

We have shown that the fundamental FCP soliton in a quadratic medium is a sech ${ }^{2}$ solitary wave, and not a wave packet. The latter are described in the frame of the $\mathrm{mKdV}$, $\mathrm{sG}$, and $\mathrm{mKdV}$-sG models by breather solutions. Although the properties of breathers are well known, we believe that it is worth recalling them for the readers that are not specialists of soliton theory. Expression (44), due to algebraic proper-

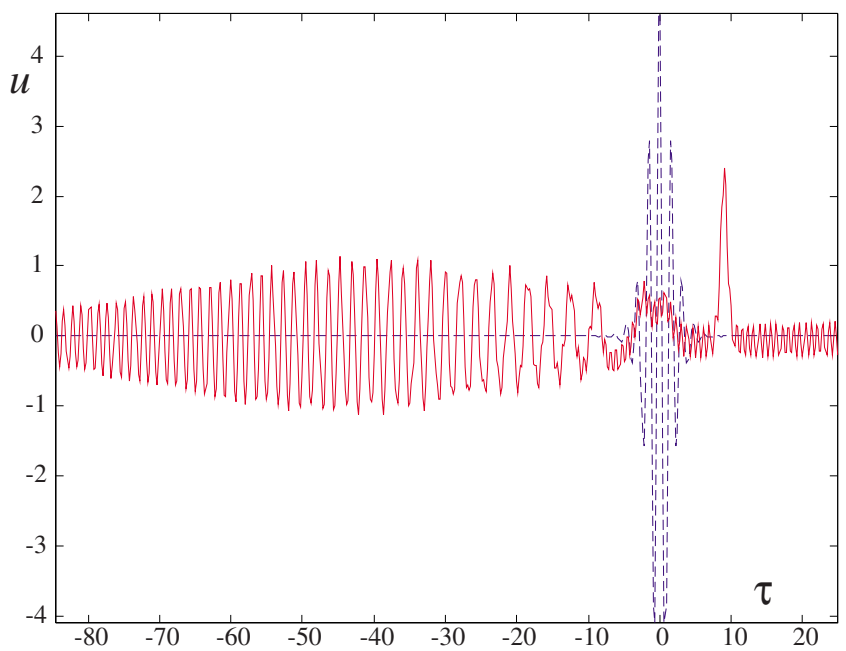

FIG. 3. (Color online) Evolution of an initial FCP into a KdV soliton and dispersive waves. The input (dashed blue line) and output (solid red line) wave profiles correspond to Fig. 2. The lines represent the electric field $E_{2}$ itself, and not an envelope. 


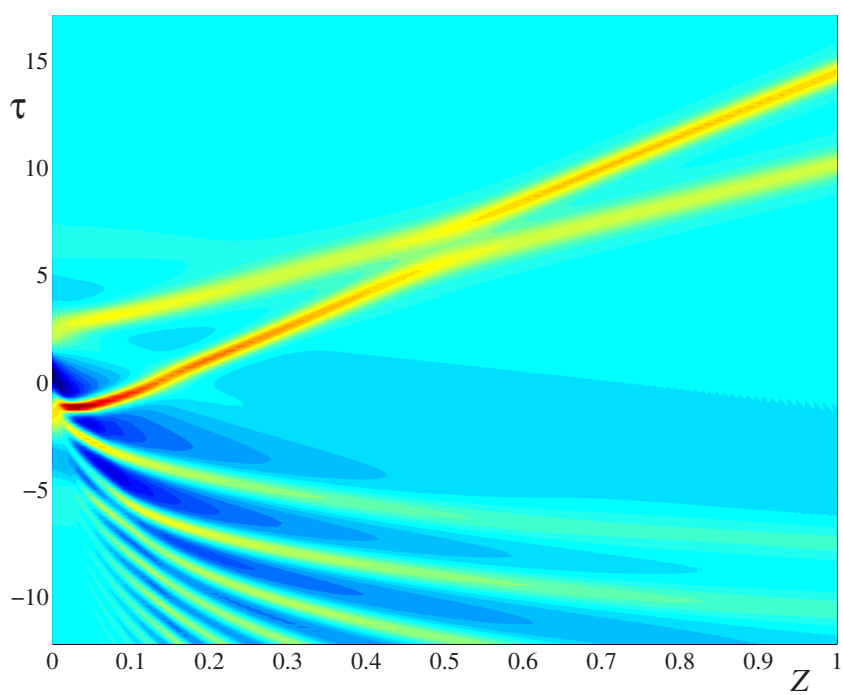

FIG. 4. (Color online) An input FCP evolves into two half-cycle solitons and radiation. The two half-cycle solitons have different velocities, higher than that of the radiation. They interact keeping their characteristics. The figure is drawn for an input $\mathrm{CE}$ phase equal to $0.8 \pi$.

ties, solves the $\mathrm{KdV}$ equation (8) for any complex value of the soliton parameter $p$. The $N$-soliton solution has the same property, and an adequate choice of the parameters allows one to build a breather solution from the two-soliton solution. However, neither realness, nor regularity, nor stability, nor robustness, is ensured this way. The breather solution to the mKdV equation [Fig. 1(b)] is regular and describes FCP solitons [8-10]. KdV also possesses breatherlike solutions, constructed by the same procedure, but these solutions are singular [28] [Fig. 1(d)].

Recall that the equivalence between $\mathrm{KdV}$ and $\mathrm{mKdV}$, due to the Miura transform [29], holds if complex solutions are considered, but not if we consider real fields. The two mKdV equations

$$
u_{t}+6 \eta u^{2} u_{x}+u_{x x x}=0,
$$

with $\eta$ equal to either +1 or -1 , cannot be reduced one to the other if $u$ is real, and the Miura transform is real for $\eta=-1$ only. The $\mathrm{mKdV}$ equation valid for FCP soliton propagation in a two-level medium [9], or more generally in a medium with focusing cubic Kerr nonlinearity, is the one with $\eta$ $=+1$, which admits real regular breathers.

It has been proven [30] that in the case of $\mathrm{mKdV}$, a breather was preferentially formed from a "symmetric" initial data, while a soliton arose if the input broke the symmetry. Hence FCP solitons (of wave-packet type) form in a cubic focusing medium, and half-cycle single-oscillation solitons are very rare. Further, the $\mathrm{mKdV}$ soliton may have both signs with equal probabilities, since Eq. (48) is invariant through $u \rightarrow-u$. Hence a train of half-cycle solitons in a cubic medium would contain as much positive solitons as negative ones, and resemble an oscillating train.

As a consequence of the nonexistence of a regular breather to $\mathrm{KdV}$, in a medium with quadratic optical nonlin- a)

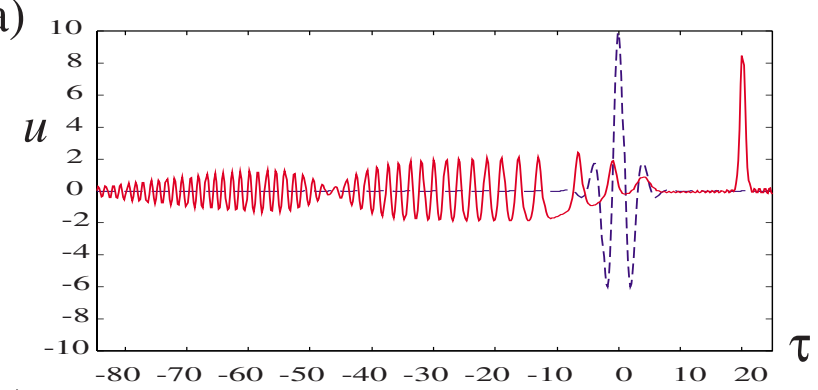

b)

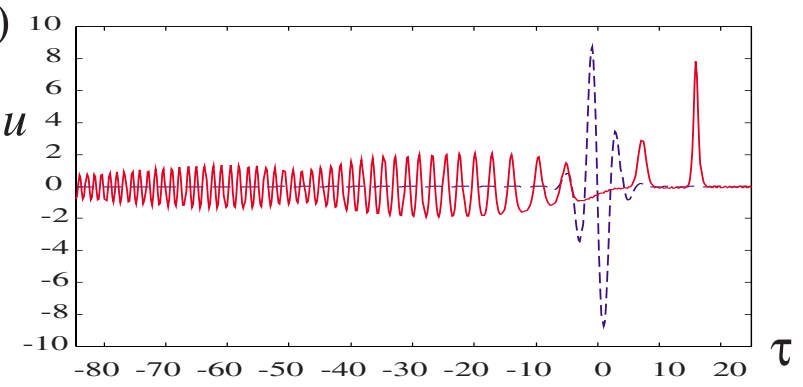

c)

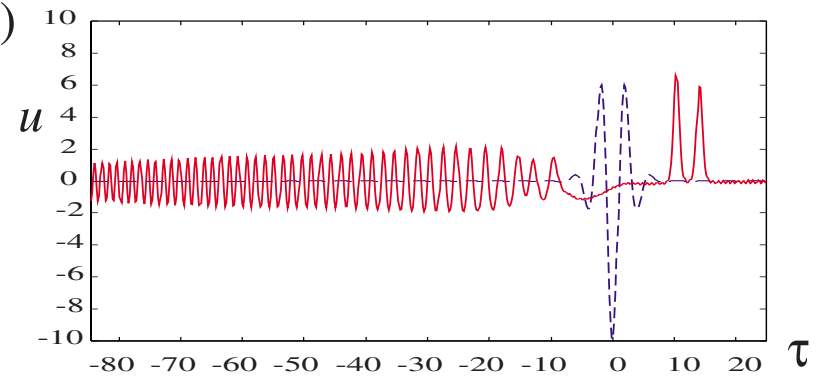

FIG. 5. (Color online) The input (dashed blue line) and output (solid red line) wave profiles for an input carrier-envelope phase equal to 0 (a), $\pi / 2$ (b), and $\pi$ (c). It is seen that the number of emitted solitons depends on the CE phase of the input FCP.

earity, the fundamental soliton systematically arises, and always has the same sign; that is, half-cycle single-oscillation solitons will be produced, and they always have the same polarity. This is closely related to the noncentrosymmetry required for quadratic nonlinearity.

Notice that the $\mathrm{mKdV}$ equation with $\eta=-1$ can describe FCP propagation in a medium with defocusing cubic Kerr nonlinearity. Since it does not admit any regular breather, the general input will decay into a train of solitons, i.e., halfcycle pulses. However, the solitons will have random sign, and the train remains close to an oscillating wave.

\section{CONCLUSION}

Half-cycle optical pulses, which are $\mathrm{KdV}$ solitons, form when a FCP is launched into a quadratic nonlinear medium. They consist of a single hump, without any satellite oscillation. They always have an absolute phase zero, in the sense that the field polarity is completely determined by the properties of the medium. As a consequence, the mean value of the optical electric field is not zero. This latter property might find applications in the frame of optical poling. 
[1] A. Scrinzi, M. Yu. Ivanov, R. Kienberger, and D. M. Villeneuve, J. Phys. B 39, R1 (2006).

[2] T. Brabec and F. Krausz, Rev. Mod. Phys. 72, 545 (2000).

[3] M. V. Tognetti and H. M. Crespo, J. Opt. Soc. Am. B 24, 1410 (2007).

[4] T. Schäfer and C. E. Wayne, Physica D 196, 90 (2004).

[5] Y. Chung, C. K. R. T. Jones, T. Schäfer, and C. E. Wayne, Nonlinearity 18, 1351 (2005).

[6] A. Sakovich and S. Sakovich, J. Phys. A 39, L361 (2006).

[7] S. A. Skobelev, D. V. Kartashov, and A. V. Kim, Phys. Rev. Lett. 99, 203902 (2007).

[8] I. V. Mel'nikov, D. Mihalache, F. Moldoveanu, and N.-C. Panoiu, Phys. Rev. A 56, 1569 (1997).

[9] H. Leblond and F. Sanchez, Phys. Rev. A 67, 013804 (2003).

[10] H. Leblond, S. V. Sazonov, I. V. Mel'nikov, D. Mihalache, and F. Sanchez, Phys. Rev. A 74, 063815 (2006).

[11] H. Mashiko, C. M. Nakamura, C. Li, E. Moon, H. Wang, J. Tackett, and Z. Changa, Appl. Phys. Lett. 90, 161114 (2007).

[12] C. R. Menyuk, R. Schiek, and L. Torner, J. Opt. Soc. Am. B 11, 2434 (1994).

[13] G. I. Stegeman, D. J. Hagan, and L. Torner, Opt. Quantum Electron. 28, 1691 (1996).

[14] A. V. Buryak and Yu. S. Kivshar, Phys. Lett. A 197, 407 (1995).

[15] R. Schiek, Y. Baek, and G. I. Stegeman, Phys. Rev. E 53, 1138
(1996).

[16] L. Torner, D. Mihalache, D. Mazilu, E. M. Wright, W. E. Torruellas, and G. I. Stegeman, Opt. Commun. 121, 149 (1995).

[17] B. A. Malomed, D. Mihalache, F. Wise, and L. Torner, J. Opt. B Quantum Semiclassical Opt. 7, R53 (2005).

[18] H. Leblond, J. Phys. A 31, 5129 (1998).

[19] L.-C. Crasovan, J. P. Torres, D. Mihalache, and L. Torner, Phys. Rev. Lett. 91, 063904 (2003).

[20] H. Leblond, Phys. Rev. Lett. 95, 033902 (2005).

[21] H. Leblond, J. Phys. B 41, 043001 (2008).

[22] Y. S. Kivshar, Opt. Lett. 16, 892 (1991).

[23] Y.-R. Shen, The Principles of Nonlinear Optics (Wiley, New York, 1984).

[24] J. Boussinesq, J. Math. Pures Appl. 17, 55 (1872).

[25] R. Hirota, in Solitons, edited by R. K. Bulough and P. J. Caudrey (Springer, Berlin, 1980), Chap. 5, pp. 157-176.

[26] C. S. Gardner, J. M. Greene, M. D. Kruskal, and R. M. Miura, Phys. Rev. Lett. 19, 1095 (1967).

[27] R. K. Dodd, J. C. Eilbeck, J. D. Gibbon, and H. C. Morris, Solitons and Nonlinear Wave Equations (Academic Press, London, 1982).

[28] M. Jaworski, Phys. Lett. 104A, 245 (1984).

[29] R. M. Miura, J. Math. Phys. 9, 1202 (1968).

[30] S. Clarke, R. Grimshaw, P. Miller, E. Pelinovsky, and T. Talipova, Chaos 10, 383 (2000). 